\title{
EL PAPEL DE LOS MARCADORES TUMORALES EN LA CONSULTA DE UROLOGÍA PARA EL SCREENING, DIAGNÓSTICO Y SEGUIMIENTO DEL CÁNCER DE VEJIGA
}

\author{
M.F. LORENZO GÓMEZ \\ Department of Urology. University of Miami. Miami. Florida, USA \\ Actas Urol Esp. 27 (2): 110-116, 2003
}

\section{RESUMEN}

"EL PAPEL DE LOS MARCADORES TUMORALES EN LA CONSULTA DE UROLOGÍA PARA EL SCREENING, DIAGNÓSTICO Y SEGUIMIENTO DEL CÁNCER DE VEJIGA"

OBJETIVO: Evaluación de la utilidad de los marcadores tumorales para el screening y el diagnóstico del cáncer transicional de vejiga.

MÉTODOS: Identificación y análisis crítico de la literatura publicada en marcadores tumorales de vejiga mediante una búsqueda en Medline. Se ha comparado la sensibilidad y especificidad de los distintos marcadores.

RESULTADOS: Los métodos convencionales no invasivos para el diagnóstico (síntomas y citología de orina) y para el screening y seguimiento del carcinoma transicional de vejiga, no deben ser considerados suficientemente sensibles. Algunos marcadores tumorales pueden ser una alternativa razonable a la citología de orina convencional.

CONCLUSIÓN: Los marcadores tumorales para el screening y el seguimiento del cáncer de vejiga han de ser considerados una alternativa a la citología. En el futuro, marcadores más sensibles y específicos deben sustituir asimismo a la cistoscopia de rutina.

PALABRAS CLAVES: Vejiga. Tumor. Marcadores. Diagnóstico. Screening.

\begin{abstract}
“THE ROLE OF TUMOR MARKERS IN OFFICE UROLOGY FOR SCREENING, DIAGNOSIS AND FOLLOW UP OF BLADDER CANCER"

PURPOSE: Review the tumor markers for screening and diagnosis of transitional cell carcinoma of the bladder.

METHODS: The published literature on bladder cancer markers was identified using a MEDLI$\mathrm{NE}$ search and critically analyzed. The sensitivity and specificity of the various markers were compared.

RESULTS: Conventional non-invasive methods for diagnosis (symptoms and urine cytology) and for screening and follow-up of transitional cell carcinoma of the bladder are not very sensitive and must not be considered sufficient. Some tumor markers seem to be a good alternative to the routine urinary cytology.

CONCLUSION: The role of tumor markers for screening and follow-up of bladder tumors as an alternative to cytology deserve consideration. In the future, more sensitive and specific markers may replace routine cystoscopy.
\end{abstract}

KEY WORDS: Bladder. Tumor. Markers. Diagnosis. Screening. 
$\mathrm{E}^{1}$ cáncer de vejiga consiste en la transformación maligna de la vejiga. Más del 90\% de los tumores vesicales son carcinomas de células transicionales (TCC) ${ }^{1}$. La totalidad del urotelio, desde la pelvis renal hasta la uretra, es susceptible de esta transformación, siendo la vejiga el sitio más frecuente de presentación. Cada año, se diagnostican aproximadamente 263.000 casos nuevos de cáncer de vejiga en el mundo, y se le atribuyen específicamente alrededor de 115.000 muertes $^{2,3}$. En contraste con Estados Unidos, la incidencia y mortalidad están aumentando en algunos países europeos, a pesar del hecho de que la mayoría de los nuevos casos se diagnostican como tumores superficiales ${ }^{4}$. En alrededor del $85 \%$ de los pacientes, el síntoma de presentación es la hematuria indolora. Actualmente, si un paciente en edad de presentación del cáncer de vejiga, tiene una hematuria inexplicable, el diagnóstico conlleva una cistoscopia y biopsia ${ }^{5}$.

Dado que la citología convencional y la citometría de flujo (ver más adelante) tienen pobre sensibilidad para tumores bien y moderadamente diferenciados, la utilización de nuevos tests/marcadores tumorales ayudarian a los tradicionales métodos de diagnóstico y de estadiaje, apoyando la indicación del tratamiento adecuado en un paciente individual y mejorando la vigilancia de aparición de las recurrencias. En este artículo se describen los nuevos métodos disponibles para mejorar el diagnóstico y el seguimiento del cáncer de vejiga.

\section{Screening}

El coste/efectividad de un screening indiscriminado para el cáncer de vejiga, dada la baja prevalencia en la población general, es poco probable. Sin embargo, el screening en individuos con alto riesgo, como los expuestos a carcinógenos conocidos (cigarrillos, ciclofosfamida, aminas aromáticas y radiación pelviana, entre otros) con unos marcadores precisos, detectaría el cáncer vesical cuando todavía está confinado en la mucosa.

Actualmente el $28 \%$ de mujeres y el $43 \%$ de hombres de la Unión Europea fuman cigarrillos ${ }^{6}$. El 35\% de todos los cánceres vesicales en mujeres y el $50 \%$ en varones se atribuyen al consumo de cigarrillos $^{6}$. Un reciente informe de la Asociación Internacional para la Investigación en Cáncer
(IARC) reveló que los fumadores tienen de 5 a 6 veces más riesgo de desarrollar cáncer de vejiga que los no fumadores ${ }^{7,8}$. El medio más seguro de eliminar el riesgo de cáncer es dejar de fumar. Sin embargo, aún dejando de fumar, el riesgo de cáncer de vejiga persiste durante varios años. Por lo tanto, habría que considerar la vigilancia de fumadores en el presente o en el pasado, así como de los trabajadores expuestos a un carcinógeno, mediante la utilización de un marcador tumoral de vejiga no invasivo para detectar el cáncer antes de que fuera invasivo. Por ejemplo, es un estudio de screening en trabajadores expuestos a benzidina, Hemstreet et al. encontraron que la sensibilidad, especificidad y odds ratio (OR) de un perfil molecular basado en DNA4CER/p300, era útil en la cohorte estudiada. Además, todos los tumores vesicales detectados por el perfil molecular fueron superficiales $^{9}$.

El screening de la hematuria no es específico para el cáncer de vejiga, y la citología tiene una baja sensibilidad, en particular para los tumores de bajo grado. Por lo tanto, si se pretende un marcador para screening de cáncer de vejiga, ha de tener alto valor predictivo positivo y un razonable valor predictivo negativo, para disminuir una ansiedad innecesaria e investigaciones caras debidos a falsos resultados positivos.

\section{Recurrencia}

La proporción de recurrencia de los tumores superficiales de vejiga después de la resección transuretral, llega hasta el 70\%. Además, del 20 al $25 \%$ de tales recurrencias presentan un grado más alto que el tumor inicial. Asimismo, hasta un $10 \%$ de los tumores superficiales progresan a invasión o a metástasis ${ }^{10}$. Actualmente, el seguimiento después de la resección transuretral implica cistoscopia y citología cada 3 meses durante los primeros 18 a 24 meses, cada 6 meses durante los siguientes 2 años y anualmente después.

Debido a la baja sensibilidad de la citología para detectar carcinomas de bajo grado, y los riesgos y molestias que la cistoscopia causa a los pacientes, se necesitan nuevos test no invasivos que monitoricen con precisión la recurrencia en el seguimiento de tratamientos conservadores de la vejiga. Los marcadores tumorales vesicales parecen ser la solución. 


\section{MARCADORES TUMORALES DE VEJ IGA EN EL DIAGNÓSTICO Y SEGUIMIENTO (Tabla I)}

Citología de Papanicolau (Pap): Actualmente la citología Pap es el marcador tumoral de vejiga no invasivo estándar. Sin embargo, aunque la citología es altamente específica y sensitiva en tumores de alto grado y carcinoma in situ de la vejiga, algunos estudios han encontrado una sensibilidad tan baja como del $17 \%$ en el grado G $1^{11}$ y del $26 \%$ en el estadio $\mathrm{Ta}^{12}$. Además, en pacientes con tumores de alto grado, la citología de orina puede resultar falso negativo hasta en un $20 \%{ }^{1}$. La citología falso positivo puede ocurrir entre un 1 y un $12 \%$ de pacientes, debido a atipia urotelial, a inflamación o a cambios causados por la radio o la quimioterapia ${ }^{13}$. Diversos autores proclaman que la citología no debe ser considerada ya más como una herramienta efectiva para el diagnóstico de TCC, y que no debe ser considerada parte del seguimiento rutinario de los pacientes diagnosticados de tumor vesical ${ }^{14}$.

\section{TABLA I}

MARCADORES TUMORALES DE VEJIGA EN DIAGNÓSTICO

\begin{tabular}{|c|c|}
\hline Marcador & Sensibilidad/ Especificidad \\
\hline Citología Papanicolau & $\begin{array}{l}33 \% / 99 \% 19 \\
35 \%-40 \%-\text { rango } 16 \% \text { a } 60 \% / 90-95 \%^{23} \\
37 \% / 70 \%{ }^{22} \\
40-47 \% \text { en promedio- } 26 \% \text { en Ta, } 64 \% \text { en } \mathrm{T} 1 \text { y más alto } / 94 \%^{12} \\
41 \% \text { tumor nuevo/94\% }{ }^{20} \\
58 \% \text { promedio- } 17 \% \text { en } \mathrm{G} 1,61 \% \text { en } \mathrm{G} 2,90 \% \text { en } \mathrm{G} 3 / 100 \%{ }^{11} \\
61 \% / 100 \% 33\end{array}$ \\
\hline HA-HAase & $\begin{array}{l}85,1 \% / 89 \% \text { en } \mathrm{G}^{23} \\
100 \% \text { en } \mathrm{G} 2-\mathrm{G} 3 / 89 \% \text { en } \mathrm{G} 2-\mathrm{G} 3{ }^{23}\end{array}$ \\
\hline Ácido hialurónico & $91,9 \% / 92,8 \%{ }^{38}$ \\
\hline Hialuronidasa & $100 \% / 88,8 \%$ en $\mathrm{G} 2-\mathrm{G} 3^{39}$ \\
\hline BTA stat & $\begin{array}{l}13 \%-48 \% \text { en } \mathrm{G} 1-36 \%-67 \% \text { en } \mathrm{G} 2-\mathrm{G} 3-80 \% \text { en } \mathrm{G} 3 / 84 \%{ }^{18} \\
55 \% / 54 \%{ }^{20} \text { en recurrencia } \\
57 \% / 68 \%{ }^{11} \\
57 \%-83 \% / 46 \%-73 \% 23 \\
65 \% / 64 \% \text { en promedio, } 46 \% \text { sin otra enfermedad genitourinaria, } 71 \% \text { sin enfermedad } \\
\text { genitourinaria }^{19}\end{array}$ \\
\hline NMP22 & $\begin{array}{l}45 \% / 64 \% \text { en recurrencia }{ }^{20} \\
47 \%-100 \% / 60 \%-70 \%{ }^{23} \\
48 \% \text { en promedio- } 52 \% \text { en } \mathrm{G} 1,45 \% \text { en } \mathrm{G} 2,50 \% \text { en } \mathrm{G} 3 / 60-70 \%{ }^{11} \\
66 \% / 70^{22} \\
72 \% \text { en promedio, } 83 \% \text { en pT1-pT2, } 55 \% \text { en pTa } / 73 \% \text { en } \text { promedio }^{21} \text { con umbral de } \\
10 \mathrm{Ul} / \mathrm{ml} \\
75 \% / 81,6 \%^{34} \\
81 \% \text { en promedio- } 81 \% \text { en pTa, } 82 \% \text { en } \mathrm{T} 1 \text { y más alto, } 69 \% \text { en } \mathrm{G} 1,86 \% \text { en } \mathrm{G} 2,93 \% \\
\text { en G3, } 67 \% \text { en CIS } / 77 \% \text { en promedio }{ }^{12} \text { con umbral de } 7 \mathrm{U} / \mathrm{ml}\end{array}$ \\
\hline Immunocyt ${ }^{\circledR}$ & $\begin{array}{l}86 \% \text { en promedio- } 84 \% \text { en } \mathrm{G} 1-90 \% \text { en } \mathrm{G} 3 / 79 \%{ }^{18} \\
86 \% / 79 \%^{23} \\
90 \% / 90 \%{ }^{20} \\
90 \% / 90 \%{ }^{24} \text { en G3 }\end{array}$ \\
\hline DD23 & $85 \% / 95 \%$ en individuos $\operatorname{sanos}^{28}$ \\
\hline $\mathrm{UBC}$ & $40 \%$ en recurrencia- $60 \%$ en nuevos tumores $/ 72 \%$ en recurrencia ${ }^{20}$ \\
\hline CYFRA21-1 & $\begin{array}{l}96 \% / 74 \%^{31} \\
32 \% / 95 \%^{32}\end{array}$ \\
\hline
\end{tabular}


Test urinario HA-HAase: Es una combinación de dos test: test del ácido hialurónico (HA) y test de la hialuronidasa (HAase). El test HA-HAase detecta HA, un glicosaminoglicano, y la Haase, su enzima degradante, en la orina. HA es un glicosaminoglicano no sulfatado presente en los tejidos y fluidos normales. La concentración tisular de HA está elevada en los tumores de colon, Wilms y de vejiga $^{15}$. Lokeshwar caracterizó la HAase asociada al tumor de vejiga, y describió la HA y HAase como marcadores para detectar el cáncer vesical y evaluar su grado. Este test se lleva a cabo a través de un procedimiento tipo ELISA, y los niveles de HA y HAase se normalizan respecto a las proteínas urinarias $^{15}$. El test HA-HAase ha mostrado una sensibilidad del 90-92\% y una especificidad del $80-84 \%$ en el diagnóstico de nuevos tumores. En la detección de tumores vesicales de bajo grado muestra una sensibilidad del $85,1 \%$ y una especificidad del $86,4 \%$, valores considerablemente superiores a la citología urinaria de rutina. La misma sensibilidad y especificidad se han encontrado en seguimiento de las recurrencias ${ }^{16}$. Un test HA (+) sugiere la presencia de cáncer de vejiga, independientemente del grado. Los niveles de HAase se relacionan con el potencial maligno del cáncer de vejiga, y son más altos en tumores de grados G2/G3. Un resultado positivo de cada test (test HA y test HAase) indicaría grado 2 o más alto, mientras que un resultado positivo del test HA, pero negativo del test Haase, indicaría un tumor de bajo grado. El test combinado HA-HAase mejora la sensibilidad al 92\% manteniendo una especificidad del $85 \%$. En tumores de vejiga de grado 2 ó 3, la sensibilidad del test fue del $100 \%$ y la especificidad del $89 \%$. Por lo tanto, la combinación de los dos test aporta información, de forma no invasiva, sobre el grado del tumor (bien grado G1, o G2 o G3). El test HA-HAase detecta la mayoría de los cánceres de vejiga, así como los tumores detectados por cada test individualmente ${ }^{16}$.

El test BTA stat mide en orina las proteínas relacionadas con el factor $\mathrm{H}$ del complemento, mediante una reacción colorimétrica antígenoanticuerpo. Estas proteínas son producidas por diversas líneas celulares de cáncer, tales como de vejiga, cervix y riñón. En comparación directa con la citologia urinaria, el test BTA stat tiene generalmente una sensibilidad más alta, pero muy baja especificidad. En los estudios iniciales, el BTA stat mostró una sensibilidad del $58 \%{ }^{17}$, sin embargo en algunas series ${ }^{18}$ se ha encontrado una sensibilidad tan baja como del 13\% para detectar tumores de grado G1. Además, puede dar hasta un $84 \%$ de falsos positivos en presencia de hematuria macroscópica, baja concentración de creatinina en orina, traumatismo genitourinario reciente (incluida una cistoscopia), tratamiento intravesical (BCG u otro), litiasis urinaria, inflamación del tracto urinario y otros procesos malignos de la vejiga. La especificidad de este test fue del $95 \%$ en voluntarios normales pero descendió hasta el $46 \%$ cuando coexistían condiciones no neoplásicas ${ }^{19} \mathrm{y}$ hasta el $28 \%$ en los dos años subsiguientes al tratamiento con $\mathrm{BCG}^{17,20}$.

El test NMP22 mide proteínas de la matriz nuclear involucradas en la replicación de DNA y en la sintesis de RNA durante la mitosis. Su presencia es común en todas las células y se detecta mediante inmunoensayo tipo sandwich ${ }^{20}$. Estableciendo un valor umbral o de corte de $10 \mathrm{U} / \mathrm{ml}$, se encontró una sensibilidad del $83 \%$ en la detección del cáncer vesical en estadios pT1 y pT2 para el test NMP22, pero sólo una sensibilidad del $55 \%$ en el estadio $\mathrm{pTa}^{21}$. Aunque algunos autores $^{22}$ han encontrado el NMP22 más sensible que la citología de orina para el seguimiento de tumores vesicales de alto riesgo $(80,9 \%$ de sensibilidad frente al 40\%), aquel muestra una especificidad más baja $(64,3 \%$ frente al $100 \%)$ y una proporción inaceptable de falsos positivos ${ }^{12,21,22}$. Estos resultados falsos positivos son consecuencia de procesos inflamatorios tales como resección transuretral, infección, cistitis, y en el caso de piuria y/o hematuria. También puede ser positivo en otras condiciones neoplásicas como en el carcinoma de células renales. Aunque los datos de sensibilidad parecian prometedores, la baja especificidad limita su aplicación. Además, en el diagnóstico y seguimiento de cáncer de vejiga de bajo grado, la citología tiene una sensibilidad más alta que el NMP22 $2^{11,20,23}$.

El test Immunocy $t^{\circledR}$ se basa en el uso de anticuerpos monoclonales M344, 19A211 y LDQ10, para identificar antígenos asociados a tumor en la superficie de las células. Se realiza mediante microscopía inmunofluorescente en la muestra de orina ${ }^{24}$. Las series iniciales encontraron un 95\% de sensibilidad para tumores de bajo estadio pero 
la sensibilidad fue menor en estudios subsiguientes. Bonner et al. encontraron una sensibilidad del $78 \%$ usando análisis de imagen fluorescente con un nivel de corte de 2 o más células positivas por 10.000 células. La especificidad fue del 94\% en individuos sanos y del $72 \%$ en pacientes con obstrucción al tracto de salida de la vejiga ${ }^{25}$. Konety encontró expresión de M344 en el 70\% de tumores superficiales de vejiga pero sólo del 15\% en tumores invasivos. Mientras que 19A211 se encontró en el 50\% de tumores vesicales invasivos y en el 60\% de casos de carcinoma in situ ${ }^{26}$. Vrisema asegura que el Immunocyt ${ }^{\circledR}$ no permite omitir la cistoscopia en el seguimiento de tumores uroteliales superficiales de vejiga de bajo grado ${ }^{27}$, mientras que otros autores informan de una sensibilidad del 90\% y una especificidad del 90\% en tumores de alto grado ${ }^{24}$.

DD23 es un antígeno asociado a tumor detectado mediante inmunocitología. La identificación del antígeno DD23 en orina en pacientes con cáncer de vejiga se ha encontrado con una sensibilidad del 85\%, y una especificidad del 95\% en individuos sanos y controles asintomáticos. Este test ahora está disponible comercialmente aunque tiene la desventaja de la sensibilidad disminuida debido a la disminución o pérdida de la expresión del antígeno durante el trasporte de la muestra por la inestabilidad de la expresión ${ }^{28}$.

El antígeno $U B C$ detecta fragmentos de citokeratina 8 y 18 en orina. Se encontró una sensibilidad del 60\% para diagnosticar nuevos TCC de vejiga, y una sensibilidad del 40\% y una especificidad del $72 \%$ para las recurrencias ${ }^{20}$. Un estudio que combinó UBC con CYFRA2 1-1 (ver más adelante) y NMP22 concluyó que cuando los resultados eran negativos, podría disminuirse la frecuencia de las cistoscopias para el seguimiento y detección de recurrencias $^{29}$. También se ha señalado que el uso combinado de DNA/citofluometría de citokeratina podría ser útil en detectar recurrencias ${ }^{30}$. La desventaja de los test basados en las citokeratinas es que no son específicos para el cáncer de vejiga, y pueden ser positivos en otro tipo de tumores epiteliales. Este hecho hace que otros marcadores tumorales más específicos sean más útiles para detectar el cáncer de vejiga.

CYFRA 21-1 detecta el fragmento soluble de la cytokeratina 19 . La citokeratina 19 se expresa en el urotelio normal y en las células tumorales vesicales. Utiliza un ensayo inmunnoradiométrico con un inmunoabsorbente ligado a enzima. Utilizando un nivel de corte triplicado a $4 \mathrm{ng} / \mathrm{ml}$ de esta citokeratina en orina. Pariente encontró una sensibilidad del 96\% y una especificidad del 74\% para detectar TCC vesical (31). Mientras que otros autores sólo encontraron una sensibilidad del 46\% para la CYFRA $21-1^{32}$.

\section{OTROS MARCADORES TUMORALES VESICALES EN EL DIAGNÓSTICO (Tabla II)}

Citometría de flujo: Mide el contenido de ADN de las células. Cuantifica la población celular aneuploide y la actividad proliferativa (porcentaje de células en fase $\mathrm{S}$ ) en un tumor ${ }^{1}$. Un estudio de revisión informó de un $72,5 \%$ de sensibilidad y un $80 \%$ de especificidad para la citometría de flujo, $62,5 \%$ de sensibilidad y $100 \%$ de especificidad para la citología, y una sensibilidad del $73 \%$ con una especificidad del 100\% para la hibridación fluorescente in situ (FISH) ${ }^{33}$. Sin embargo, las células inflamatorias pueden formar fracciones celulares hiperdiploides que hacen muy difícil la interpretación de la citometría de flujo estándar ${ }^{1}$.

El test Quanticyt ${ }^{\circledR}$ se basa en un análisis cariométrico de imágenes al microscopio óptico de las células obtenidas por lavados vesicales, estudiando la forma del núcleo celular y el contenido de ADN para atribuir un grado a la muestra del lavado vesical. Wiener encontró una sensibilidad del 59\% y una especificidad del 93\% para el Quanticyt ${ }^{\circledR}{ }^{11}$, mientras que Witjes encontró solamente una sensibilidad del 45,4\% y una especificidad del 70,6\%34.

\section{TABLA II}

OTROS MARCADORES TUMORALES DE VEJIGA PARA DIAGNÓSTICO

\begin{tabular}{||l|l|}
\hline Marcador & Sensibilidad/ Especificidad \\
\hline Citometría de flujo & $72 \% / 80 \%^{33}$ \\
\hline Quanticyt $^{\circledR}$ & $45,4 \% / 70,6 \%^{34}$ \\
& $59 \% / 93 \%^{11}$ \\
& $60 \%-70 \% / 70 \%^{23}$ \\
\hline Telomerasa & $62 \% / 96,4 \%^{35}$ \\
& $80 \% / 80 \%^{12}$ \\
\hline BLCA-4 & $96,4 / 100^{26}$ \\
\hline CD44 & $77 \% / 10 \%^{37}$ \\
\hline
\end{tabular}


Este test requiere técnicos expertos y un sistema de análisis de imagen caro, y sólo se realiza en especímenes del lavado vesical, necesitando un número relativamente grande de células. Por lo tanto, de momento no se ha establecido su aceptación y disponibilidad para detectar el cáncer de vejiga.

La telomerasa es una secuencia de nucleótidos en el extremo 3' de la cadena enrollada de ADN que permanece libre después de cada ciclo de replicación. La telomerasa no está presente en la mayoría de las células somáticas normales, y la medición mediante RT-PCR (reacción en cadena de la polimerasa y retrotranscripción) de la transcriptasa inversa de la subunidad catalítica de la telomerasa humana (hTERT) en las células exfoliadas de la orina, se ha propuesto como marcador para detectar el cáncer vesical ${ }^{35}$. Aunque al principio se encontró una sensibilidad del $85 \%$ para la telomerasa comparada con una sensibilidad del 50\% para la citología ${ }^{12}$, en un estudio reciente la sensibilidad para detectar cáncer de vejiga con telomerasa con un ensayo de amplificación repetida de la telomerasa en muestras de orina ha sido tan baja como del $7 \%{ }^{36}$.

El test BLCA-4 detecta la proteína de la matriz celular BLCA-4 en la orina mediante inmunoensayo. Konety propuso la determinación de BLCA4 en la orina para el screening del cáncer de veji$\mathrm{ga}^{26}$. Sin embargo este test no se ha investigado de forma fehaciente en pacientes con cáncer de vejiga para dar resultados concluyentes.

CD44 es una molécula de adhesión de la superficie celular con diferentes isoformas, que se sobreexpresa en diversos cánceres humanos. Un estudio encontró una sensibilidad del $77 \%$ y una especificidad del 100\% para detectar cáncer de vejiga $^{37}$. Sin embargo la desventaja de este test es que las muestras de orina deben ser procesadas inmediatamente mediante RT-PCR, lo cual no siempre es posible.

\section{CONCLUSIONES}

Actualmente no están establecidos protocolos para el screening del carcinoma urotelial en la población de riesgo, como la expuesta a carcinógenos. Sin embargo, y teniendo también en cuenta razones éticas, como en el caso de los carcinógenos industriales u ocupacionales, debería esta- blecerse la utilización de un marcador preciso, o una combinacion de ellos, para prevenir el desarrollo de tumores invasivos. Aunque algunos autores han aportado resultados alentadores, se necesitan más estudios en este área.

Por otra parte, para el diagnóstico y el seguimiento después de la resección transuretral, los marcadores tumorales ya están disponibles. Sin embargo, deben utilizarse con mucha cautela, teniendo siempre en cuenta las condiciones del paciente (estados inflamatorios, infecciones, etc.) y las características del tumor (grado, estadio). A la luz de los conocimientos actuales, parece apropiado intentar una combinación de marcadores, como serían la citología y otro marcador cuya determinación no dependa del número de células detectables en la orina. Este marcador podría ser el test HA-HAase, dado que ha mostrado una sensibilidad del 90-92\% con una especificidad del 80$84 \%$. Se requieren estudios multicéntricos que incluyan un amplio número de pacientes para confirmar esta propuesta.

\section{REFERENCIAS}

1. EDWARD M, MESSING, CATALONA W.: Bladder cancer. Campbell's Urology. Seventh Edition 1998; 3: 2.3292.410 .

2. PATTON SE, CRAIG-HALL M, OZEN H.: Bladder cancer. Curr Opin Oncol 2002; 14 (3): 265-272.

3. PISANI P, PARKIN DM, FERLAY J.: Estimates of the worldwide mortality from eighteen major cancers in 1985. Implications for prevention and projections of future burden. Int $J$ Cancer 1993; 55: 891-903.

4. NAWROCKI S, SKACEL T, SKONECZNA I.: Pharmacotherapy of bladder cancer - practice and prospects. Expert Opin Pharmacother 2002; 3 (6): 671-679.

5. MESSING EM, VALENCOURT A.: Hematuria screening for bladder cancer. $J$ Occup Med 1990; 32: 838.

6. ZEEGERS MP, GOLDBOHM RA, VAN DEN BRANDT PA.: A prospective study on active and environmental tobacco smoking and bladder cancer risk. Cancer Causes Control 2002; 13 (1): 83-90.

7. SANKARANARAYANAN R.: Integration of cost-efective early detection programs into the health services of developing countries. Cancer 2000; 89 (3): 475-481.

8. ACS News Today: Scientistics confirm cancer risk from second-hand smoke increases cancer risk by one-fifth. Artcle date: 2002/07/09.

9. HEMSTREET GP $3^{\text {rd }}$, YIN S, MA $Z$, BONNER RB, BI W, RAO JY, ZANG M, ZHENG Q, BANE B, ASAL N, LI G, FENG P, HURST RE, WANG W.: Biomarker risk assessment and bladder cancer detection in a cohort exposed to benzidine. J Natl Cancer Inst 2001; 93 (6): 427436.

10. WOLF HK, STOBER C, HOHENFELLNER R, LEISSNER J.: Prognostic value of p53, p21/WAF1, Bcl-2, Bax, Bak and Ki-67 immunoreactivity in pT1G3 urothelial bladder carcinomas. Tumor Biol 2001; 22: 328-336. 
11. WIENER HG, MIAN C, HAITEL A, PYCHA A, SCHATZL G, MARBERGER M.: Can urine boung diagnostic tests replace cystoscopy in the management of bladder cancer? J Urol 1999; 159: 1.876.

12. LANDMAN J, CHANG Y, KAVALER E, DROLLER MJ, LIU BC.: Sensitivity and specificity of NMP22, telomerase, and BTA in the detection of human bladder cancer. Urology 1998; 52 (3): 398-402.

13. KOSHIKAVA T, LEYH H, SCHENCK U.: Difficulties in evaluating urinary specimens after local mitomycin therapy of bladder cancer. Diagn Cytopathol 1989; 5: 117.

14. DE VERE ,WHITE R.: Editorial comment. J Urol 2002; 167: 83 .

15. LOKESHWAR VB, OBEK C, PHAM HT, WEI D, YOUNG MJ, DUNCAN RC, SOLOWAY MS, BLOCK NL.: Urinary hyaluronic acid and hyaluronidase: markers for bladder cancer detection and evaluation of grade. $J$ Urol 2000; 163 (1): 348-356.

16. LOKESHWAR V, SOLOWAY MS.: Letters to the Editor. $J$ Urol 2002; 167: 1.405-1.408.

17. SAROSDY MF, HUDSON MA, ELLIS MJ, SOLOWAY MS, DE VERE WHITE R, SHEINFELD J, JAROWENKO MV, SCHELLHAMMER PF, SCHERVISH EW, PATEL JV, CHODAK GW, LAMM DL, JOHNSON RD, HENDERSON M, ADAMS G, BLUMENSTEIN BA, THOELKE KR, PFALZGRAF RD, MURCHISON HA, BRUNELLE SL: Improved detection of recurrent bladder cancer using the Bard BTA stat test. Urology 1997; 50 (3): 349-353.

18. NASUTI JF, GOMELLA LG, ISMIAL M, BIBBO M.: Utility of the BTAstat test kit for bladder cancer screening. Diagn Cytopathol 1999; 21: 27.

19. LEYH H, MARBERGER M, CONORT P, STERNBERG C, PANSADORO V, PAGANO F, BASSI P, BOCCON-GIBOD L, RAVERY V, TREIBER U, ISHAK L: Comparison of BTA stat test with voided urine cytology and bladder wash cytology in the diagnosis and monitoring of bladder cancer. Eur Urol 1999; 35 (1): 52-56.

20. BOMAN H, HEDELIN H, HOLMANG S.: Four bladder tumor markers have a disppointingly low sensitivity for small size and low grade recurrence. $J$ Urol 2002; 167: 80-83.

21. OGE O, ATSU N, KENDI S, OZEN H.: Evaluation of nuclear matrix protein 22 (NMP22) as a tumor marker in the detection of bladder cancer. Int Urol Nephrol 2001; 32 (3): 367-370.

22. OHTANI M, IWASAKI A, SHIRAIWA H: Urinary tumor marker for urothelial cancer. Gan To Kagaku Ryoho 2001; 28 (12): 1.933-1.937.

23. LOKESHWAR VB, SOLOWAY M.: Current bladder tumor test: does their projected utility fulfill clinical necessity? J Urol 165 (4): 1.067-1.077.

24. ÁLVAREZ-KINDELAN J, LÓPEZ-BELTRÁN A, REQUENA-TAPIA MJ.: Biología molecular en el cáncer vesical. Actas Urol Esp 2000; 24 (8): 604-625.

25. BONNER RB, HEMSTREET GP $3^{\text {rd }}$, FRADET Y, RAO JY, MIN KW, HURST RE.: Bladder cancer risk assessment with quantitative fluorescence image analysis of tumor markers in exfoliated bladder cells. Cancer 1993; 72 (8): 2.461-2.469.

26. KONETY BR, GETZENBERG RH.: Urine based markers of urological malignancy. J Urol 2001; 165 (2): 600-611.

27. VRIESEMA JL, ATSMA F, KIEMENEY LA, PEELEN WP, WITJES JA, SCHALKEN JA.: Diagnostic efficacy of the ImmunoCyt test to detect superficial bladder cancer recurrence. Urology 2001; 58 (3): 367-371.
28. BONNER RB, LIEBERT M, HURST RE, GROSSMAN HB,

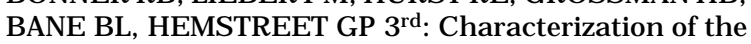
DD23 tumor-associated antigen for bladder cancer detection and recurrence monitoring. Marker Network for bladder cancer. Cancer Epidemiol Biomarkers Prevent 1996; 5 (12): 971-978.

29. SÁNCHEZ-CARBAYO M, URRUTIA M, GONZÁLEZ DE BUITRAGO JM, NAVAJO JA.: Utility of serial urinary tumor markers to individualize intervals between cystoscopies in the monitoring of patients with bladder carcinoma. Cancer 2001; 92 (11): 2.820-2.828.

30. SÁNCHEZ-CARBAYO M, CIUDAD J, URRUTIA M, MAVAJO JA, ORFAO A.: Diagnostic performance of the urinary bladder carcinoma antigen ELISA test and a multiparametric DNA/cytokeratin flowcytometry in urine voided samples from patients with bladder carcinoma. Cancer 2001; 92 (11): 2.811-2.819.

31. PARIENTE JL, BORDENAVE L, MICHEL P, LATAPIE MJ, DUCASSON D, LE GUILLON M: Initial evaluation of CYFRA 21-1 diagnostic performances as a urinary marker in bladder transitional cell carcinoma. J Urol 1997; 158 (2): 338-341

32. MADY EA.: Cytoketarins as serum markers in egyptian bladder cancer. A comparison of CYFRA 21-1, TPA and TPS. Int J Biol Markers 2001; 16 (2): 130-135.

33. CAJULIS RS, HAINES GK $3^{\text {rd }}$, FRIAS-HIDVEGI D, MCVARY K, BACUS JW.: Cytology, flow cytometry, image analysis and interphase cytogenetics by fluorescence in situ hybridization in the diagnosis of transitional cell carcinoma in bladder washes: a comparative study. Diag Cytopathol 1995; 13 (3): 214-223.

34. WITJES JA, VAN DER POEL HG, VAN BALKEN MR, DEBRUYNE FM, SCHALKEN JA: Urinary NMP22 and kariometry in the diagnosis and follow up of patients with superficial bladder cancer. Eur Urol 1998; 33 (4): 387-391.

35. YOSHIDA K, SUGINO T, TAHARA H, WOODMAN A, BOLODEKU J, NARGUND V, FELLOW SG, GOODISON S, TAHARA E, TARIN D.: Telomerase activity in bladder carcinoma and its implication for noninvasive diagnosis by detection exfoliated cancer cells in urine. Cancer 1997; 79 (2): 362-369.

36. MULLER M, KRAUSE H, HEICAPPELL R.: Comparison of human telomerase activity in urine for diagnosis of bladder cancer. Clin Cancer Res 1998; 4: 1.949.

37. MIYAKE H, HARA I, GOHJI K et al.: Urinary cytology and competitive reverse transcriptase-polimerase chain reaction analysis of a specific CD44 variant to detect and monitor bladder cancer. J Urol 1998; 160: 2.004.

38. LOKESHWAR VB, OBEK C, SOLOWAY MS, BLOCK NL: Tumor-associated hyaluronic acid: a new sensitive and specific urine marker for bladder cancer. Cancer Res 1997; 57 (4): 773-777.

39. PHAM HT, BLOCK NL, LOKESHWAR VB.: Tumor-derived hyaluronidase: a diagnostic urine marker for highgrade bladder cancer. Cancer Res 1997; 57 (4): 778783.

\footnotetext{
Dra. M.F. Lorenzo Gómez

Departmento of Urology

P.O. Box 016960 (M814)

Miami, Florida 33101
}

(Trabajo recibido el 20 septiembre de 2002) 